\title{
Auswirkungen der COVID-19-Pandemie auf die klinische Versorgung, Arbeitsprozesse und Mitarbeitenden in der Universitätsmedizin: Ergebnisse einer Interviewstudie am UKE Impact of COVID-19-pandemic on Clinical Care, Work Flows and Staff at a University Hospital: Results of an Interview-study at the UKE
}

\author{
Autoren \\ Martin Härter*1D, Daniel Bremer*1D, Martin Scherer², Olaf von dem Knesebeck ${ }^{3}$, Uwe Koch-Gromus ${ }^{1}$
}

Institute

1 Institut und Poliklinik für Medizinische Psycholgie, Center for Health Care Research, Universitätsklinikum HamburgEppendorf, Hamburg

2 Institut und Poliklinik für Allgemeinmedizin, Center for Health Care Research, Universitätsklinikum HamburgEppendorf, Hamburg

3 Institut für Medizinische Soziologie, Center for Health Care Research, Universitätsklinikum Hamburg-Eppendorf, Hamburg

Schlüsselwörter

COVID-19, Pandemie, Gesundheitsversorgung, qualitative Befragung, Qualität

Key words

COVID-19, Pandemic, Health care, Qualitative survey, Quality

Online publiziert: 21.8 .2020

\section{Bibliografie}

Gesundheitswesen 2020; 82: 676-681

DOI 10.1055/a-1226-6828

ISSN $0941-3790$

(c) 2020. Thieme. All rights reserved.

Georg Thieme Verlag KG, Rüdigerstraße 14,

70469 Stuttgart, Germany

Korrespondenzadresse

Prof. Dr. Dr. Martin Härter

Institut und Poliklinik für Medizinische Psychologie

Universitätsklinikum Hamburg-Eppendorf

20246 Hamburg

m.haerter@uke.de

\section{ZUSAMMENFASSUNG}

Ziel der Studie Die Studie beschreibt die Auswirkungen der COVID-19-Pandemie mit Blick auf Bereiche mit und ohne spezifische Versorgung von COVID-19-Patientinnen und -Patienten aus Sicht der Klinikdirektorinnen und -direktoren am Universitätsklinikum Hamburg-Eppendorf (UKE).

Methodik Im Zeitraum vom 30. April bis 12. Mai 2020 wurden mit den im UKE verantwortlichen Klinikdirektorinnen und -direktoren Interviews per Telefon, Video bzw. face-to-face mithilfe eines teilstandardisierten Fragebogens durchgeführt. Die standardisierten Antworten wurden deskriptiv analysiert. Freitextantworten wurden einer qualitativen Inhaltsanalyse unterzogen. Die entwickelten Kategorien wurden quantitativ nach Häufigkeiten analysiert.

Ergebnisse Alle leitenden Ärztinnen und Ärzte mit einer Verantwortung für die stationäre und ambulante Versorgung am UKE konnten für die Interviewstudie gewonnen werden $(\mathrm{N}=38)$. Diese berichten von gravierenden Veränderungen in der medizinischen Versorgung, v. a. im Bereich der Bettenbelegung, der Patientenzusammensetzung, den klinischen und Arbeitsprozessen sowie den diagnostischen und Behandlungsprozessen. Zusätzlich mussten viele neue Regelungen getroffen werden, um den Präventions-, Behandlungs- und Nachsorgebedarf von Patientinnen und Patienten sowie den Schutz der Mitarbeitenden zu gewährleisten. In den meisten klinischen Bereichen zeigte sich stationär wie ambulant phasenweise eine starke Reduktion in den Belegungszahlen und der Arbeitsauslastung. Teilweise stiegen aber auch Arbeitsaufwände, z. B. durch die Anpassung von Arbeitsabläufen, veränderte Kommunikations- und Personalstrukturen sowie zusätzliche Hygienemaßnahmen. Viele Befragte äußerten sich positiv über den effizienten Aufbau einer digitalen Kommunikationsstruktur. Das Personal war durch die Pandemie an sich, aber auch durch die damit verbundenen Maßnahmen vielseitig gefordert.

Schlussfolgerung Die Ergebnisse helfen, die Auswirkungen der aktuellen COVID-19-Pandemie auf die Versorgung und Arbeitsprozesse besser zu verstehen. Zudem können sie dabei unterstützen, zukünftig Maßnahmen für pandemische Krisensituationen noch besser anzupassen. Weitere Studien könnten untersuchen, wie die Mitarbeitenden unterhalb der Leitungsebene diese Krise und ihre Auswirkungen erlebt haben.

Martin Härter und Daniel Bremer teilen sich die Erstautorenschaft 


\section{ABSTRACT}

Objectives The study investigates the impact of the COVID-19 pandemic on health services with and without specific health care of COVID-19 patients through the eyes of leading physicians at the University Medical Center Hamburg-Eppendorf (UKE).

Methods From April 30 to May 12, 2020, four interviewers conducted 38 expert interviews via telephone, video or faceto-face by using a semi-standardized questionnaire. The standardized answers were analysed descriptively. The free textanswers were subject to a qualitative content analysis. The categories were analysed via quantitative frequency distributions.

Results All chief physicians with responsibility for inpatient and outpatient health care at the UKE took part in this study $(\mathrm{N}=38)$. The leading physicians reported numerous changes regarding occupancy in the hospital, patient composition, work flows and diagnostic as well as therapeutic measures. Additio- nally, various arrangements were necessary to cover the needs of prevention, treatment and follow-up care as well as protection of staff. Measures showed, on the one hand, a strong reduction in occupancy and workload in most inpatient and outpatient clinics. On the other hand, the amount of work also increased by fundamental transitions of work flows, communication, staff structure and hygiene measures. Many respondents commented positively on the rapid and efficient setup of a digital communication structure. Partially, staff was strained by the pandemic itself and by the associated measures.

Conclusion The results of the study help to understand and assess the effects of the pandemic on health care, work flows and staff. The findings may support the specification and adaptation of prospective measures and processes for pandemic crisis situations. Future studies should investigate how staff beneath the highest executive level experienced and evaluated this crisis and consequences.

\section{Hintergrund}

Ende Januar 2020 wurde der erste deutsche COVID-19-Patient in Bayern erkannt, am 26. Februar 2020 die ersten Infektionen in Baden-Württemberg und in Nordrhein-Westfalen bestätigt. Seither hat sich die COVID-19-Pandemie weltweit rasant ausgebreitet, mit 212.022 bestätigten Infizierten in Deutschland (Stand 5. August 2020). Zeitgleich zum gesellschaftlichen „Lock down“ Mitte März 2020 wurden aufgrund der COVID-19-Pandemie zahlreiche Aufbau-, Umbau- und Schutzmaßnahmen in der Gesundheitsversorgung implementiert [1-3]. Am 12. März 2020 forderte das Bundesgesundheitsministerium (BMG) alle deutschen Krankenhäuser auf, zusätzliches Personal zu gewinnen und zunächst alle planbaren Operationen und Eingriffe zu verschieben [4]. In der Folge wurden national und regional primär elektive Eingriffe und Vorsorgeuntersuchungen verschoben oder abgesagt, um Behandlungskapazitäten zu schaffen [5,6]. Durch eine Verordnung des BMG wurde darüber hinaus die Meldung freier Intensivbetten am 6. April 2020 zur Pflicht [7]. Zudem wurden stationäre Behandlungsplätze in infektiologische oder intensivmedizinische Betten umgewandelt. Schließlich wurden Hygienemaßnahmen verschärft, Besucherregelungen verändert und Behandlungsangebote sowie -prozesse angepasst [8]. Die in Hamburg zuständige Behörde für Gesundheit und Verbraucherschutz verordnete in der Pandemie eine Vorhaltung von $25 \%$ der Intensivpflegekapazität der Plankrankenhäuser und eine Freihaltung von $10 \%$ der Betten der Normalpflege der Plankrankenhäuser in der Somatik.

Bislang gab es keine systematische Untersuchung zu Auswirkungen der Pandemie in der Gesundheitsversorgung in einem universitären Klinikum, v. a. auch in den Bereichen der Versorgung, die außerhalb der unmittelbaren Behandlung und Versorgung von COVID-19-Patientinnen und -Patienten eingebunden sind [9]. Die vorliegende Studie hat das Ziel, die Auswirkungen mit Blick auf Versorgungsbereiche mit und ohne spezifische Versorgung von COVID-19-Betroffenen aus Sicht aller für die stationäre, tagesklinische bzw. ambulante Versorgung verantwortlichen Klinikdirekto- rinnen und -direktoren am Universitätsklinikum Hamburg-Eppendorf (UKE) zu beschreiben.

\section{Situation in Hamburg und am Universitätsklinikum Hamburg-Eppendorf zum Studienzeitpunkt}

Das UKE hat als großes Universitätsklinikum mehr als 13000 Mitarbeiterinnen und Mitarbeiter. Die Anzahl der Betten liegt beim UKE bei mehr als 1700 [10]. Im Jahr 2019 hat das UKE insgesamt mehr als eine halbe Million Patientinnen und Patienten versorgt. Das UKE verfügt über 13 spezialisierte medizinische Zentren mit 77 Kliniken, Polikliniken und Instituten [10].

Am 30.4.2020 gab es insgesamt 4877 COVID-19-Fälle in Hamburg, am 12.05.2020 waren es 5001 Fälle [11]. Die Anzahl der neuen COVID-19-Fälle pro Tag lag im Erhebungszeitraum (30. April bis 12. Mai 2020) zwischen 0-13 [11]. Die Behörde für Gesundheit und Verbraucherschutz teilte am 30.04.20 mit, dass 174 Personen aus Hamburg aufgrund einer Erkrankung mit COVID-19 in stationärer Behandlung waren, davon wurden 64 Personen intensivmedizinisch betreut. Am 13.05.20 berichtete die BGV, dass 100 Personen in stationärer Behandlung waren, davon wurden 40 Personen intensivmedizinisch betreut [12].

Der erste Corona-Patient wurde am 27. Februar 2020 UKE aufgenommen. Im Zuge der Pandemie wurden am UKE relevante Umstrukturierungen vorgenommen, so wurden vier COVID-19-Stationen und 2 Quarantänestationen sowie 44 zusätzliche Intensivbetten (von 128 auf 172 Betten) aufgebaut sowie eine Corona Task Force gebildet. Im Studienzeitraum waren im UKE durchschnittlich 38-45 Patientinnen und Patienten mit COVID-19 in stationärer Behandlung, von denen 18-24 intensivmedizinisch betreut wurden. 


\section{Methodik}

\section{Studiendesign, Datenerhebung und -auswertung}

Um die Auswirkungen der COVID-19-Pandemie mit Blick auf Versorgungsbereiche mit und ohne spezifische Versorgung von COVID-19-Betroffenen zu untersuchen, wurde eine Querschnittsuntersuchung mittels semistandardisierter Interviews durchgeführt. Anfang April 2020 wurde der Vorstand des UKE und das Kollegium, d. h. das Gremium aller Klinik- und Institutsdirektorinnen und -direktoren, über die Studie informiert. Dabei wurde zugesichert, dass keine personenbezogenen Daten erfasst werden. Anschließend wurden alle Klinikdirektorinnen und -direktoren mit klinischer stationärer, teilstationärer und/oder ambulanter Verantwortung der Bereiche Innere Medizin (Gastroenterologie, Onkologie, Nephrologie, Kardiologie), operative Medizin (alle Fächer), Gynäkologie und Geburtshilfe, Kindermedizin inkl. pädiatrische Onkologie, Anästhesie und Intensivmedizin, Radiologie inklusive Neuroradiologie und Strahlentherapie, Neurologie, Psychiatrie (Erwachsene sowie Kinder und Jugendliche) und Psychosomatik, Hals-Nasen-Ohren-Heilkunde (inkl. Phoniatrie), Dermatologie und Augenheilkunde sowie Zahnmedizin per Email zur Interviewstudie eingeladen.

Alle leitenden Ärztinnen und Ärzte erklärten ihre Bereitschaft zur Teilnahme und wurden telefonisch, per Video oder persönlich im Zeitraum vom 30. April bis 12. Mai 2020 interviewt. Die Daten

- Tab. 1 Stichprobenbeschreibung.

\begin{tabular}{|c|c|c|}
\hline Bereiche und Dimensionen & Häufigkeit & Prozent \\
\hline Anzahl der Befragten & 38 & \\
\hline \multicolumn{3}{|l|}{ Geschlecht der Befragten } \\
\hline männlich & 32 & 84,2 \\
\hline weiblich & 6 & 15,8 \\
\hline \multicolumn{3}{|l|}{ Zentrumszugehörigkeit } \\
\hline Kopf- und Neurozentrum & 6 & 15,8 \\
\hline Universitäres Herz- und Gefäßzentrum & 4 & 10,5 \\
\hline Zentrum für Anästhesiologie und Intensivmedizin & 2 & 5,3 \\
\hline $\begin{array}{l}\text { Zentrum für Geburtshilfe, Kinder- und } \\
\text { Jugendmedizin }\end{array}$ & 4 & 10,5 \\
\hline Zentrum für Innere Medizin & 4 & 10,5 \\
\hline Zentrum für Onkologie & 4 & 10,5 \\
\hline Zentrum für Operative Medizin & 6 & 15,8 \\
\hline Zentrum für Psychosoziale Medizin & 2 & 5,3 \\
\hline Zentrum für Radiologie und Endoskopie & 3 & 7,9 \\
\hline Zentrum für Zahn-, Mund- und Kieferheilkunde & 3 & 7,9 \\
\hline \multicolumn{3}{|l|}{ Setting } \\
\hline stationär & 34 & 89,5 \\
\hline ambulant & 37 & 97,4 \\
\hline Tagesklinik & 17 & 44,7 \\
\hline \multicolumn{3}{|l|}{$\begin{array}{l}\text { Persönlich in Diagnostik von COVID-19- } \\
\text { Patienten involviert }\end{array}$} \\
\hline ja (inkl. Screening) & 17 & 44,7 \\
\hline nein & 21 & 55,3 \\
\hline \multicolumn{3}{|l|}{$\begin{array}{l}\text { Persönlich in Behandlung von COVID-19- } \\
\text { Patienten involviert }\end{array}$} \\
\hline ja (inkl. konsiliarischer Tätigkeit) & 15 & 39,5 \\
\hline nein & 23 & 60,5 \\
\hline
\end{tabular}

wurden mithilfe eines teilstandardisierten Interviewleitfadens erhoben. Der Leitfaden enthielt insgesamt 19 bis maximal 28 Fragen (je nach Verantwortung für die verschiedenen Versorgungsettings), die offen oder geschlossen beantwortet werden konnten. Sie wurden von vier Interviewern (MH, MS, OvdK, UK) durchgeführt, wobei jeder Interviewer 9 bis 10 Interviews realisierte. Die Interviews dauerten zwischen 20-30 Minuten. Der Leitfaden wurde vor dem Einsatz mit drei Klinikleitern im Hinblick auf die Vollständigkeit und Verständlichkeit getestet. Die Autoren haben sich für die Leitfadenerstellung inhaltlich am Framework „Outcomes for Implementation Research “von Proctor et al. orientiert (Akzeptanz, Aneignung, Angemessenheit, Machbarkeit und Kosten von Maßnahmen) [13]. Der Interviewleitfaden adressierte folgende Themen:

(1) Auswirkungen auf die (teil-)stationäre und ambulante Belegung, Patientenzusammensetzung, klinischen und organisatorischen Prozesse sowie Behandlungsangebote,

(2) Regelungen zur Abdeckung von Prävention, Behandlung und Nachsorge bei Patientinnen und Patienten und zum Schutz der Mitarbeitenden,

(3) Entwicklung neuer Formate für Behandlungsangebote,

(4) Auswirkungen auf das Personal und die interdisziplinäre Zusammenarbeit.

Die standardisierten Antworten wurden deskriptiv analysiert, die Freitexte einer qualitativen Inhaltsanalyse unterzogen [14]. Entsprechend wurden die Antworten auf die offenen Fragen je Frage thematisch zu Kategorien zusammengefasst. Die Kategorien wurden induktiv abgeleitet. Die konsentierten Kategorien wurden quantitativ im Sinne von Häufigkeitsauswertungen analysiert. Kategorien, die weniger als 5 Nennungen aufweisen, werden nicht berichtet.

\section{Stichprobe}

Es wurden insgesamt 38 Personen befragt, von denen sechs Frauen sind ( $\triangleright$ Tab. 1). In der Mehrzahl handelt es sich um Klinikdirektorinnen und -direktoren bzw. wenige leitende Ärztinnen und Ärzte (mit Chefarztstatus). Zur besseren Lesbarkeit verwenden wir im Folgenden den Begriff „leitende Ärztinnen und Ärzte“. Sie repräsentieren 38 Kliniken, die in 10 unterschiedlichen UKE-Zentren verortet sind und über stationäre und/oder ambulante Bereiche und/ oder eine Tagesklinik verfügen. 45 bzw. 40 \% der leitenden Ärztinnen und Ärzte gab an, derzeit persönlich in die Diagnostik bzw. die Behandlung von COVID-19-Patientinnen und -Patienten involviert zu sein.

\section{Ergebnisse}

\section{Belegung, Patienten, klinische Prozesse und Versorgung}

\section{Belegung}

30 von 34 leitenden Ärztinnen und Ärzten, die für einen stationären Bereich verantwortlich sind, gaben an, dass die Bettenbelegung in ihren Verantwortungsbereichen deutlich verringert wurde. Die mittlere Reduktion lag bei ca. 50-60\%, sie schwankte zwischen 20 und $80 \%$. Drei (von 34; 8,8\%) Kliniken hatten ihnen zugeordnete 
> Tab.2 Auswirkungen auf klinische und organisatorische Prozesse im stationären Bereich.

\begin{tabular}{|c|c|}
\hline Kategorien & Beispiele \\
\hline Kommunikation $\left(\mathrm{N}^{*}=14\right)$ & $\begin{array}{l}\text { Geringere Anzahl von Besprechungen, kleinere Besprechungsrunden, Umstellung auf Videokonferenzen, feste Zuteilung } \\
\text { von Räumen für Teams, geringere Einbindung von anderen Disziplinen }\end{array}$ \\
\hline Personal $(\mathrm{N}=11)$ & $\begin{array}{l}\text { Trennung des Personals in zwei oder mehr (kleinere) Gruppen (Teams), ein Teil des Personals im Home-Office, } \\
\text { notwendige Umsetzung von Quarantänemaßnahmen }\end{array}$ \\
\hline $\begin{array}{l}\text { Patientinnen und } \\
\text { Patienten }(\mathrm{N}=10)\end{array}$ & $\begin{array}{l}\text { Besuchsverbot für Angehörige, Ausgangsbeschränkungen, kürzere Liegedauer, Verzögerungen bei Entlassung (erneutes } \\
\text { Testen, Weiterbetreuung), aufwändigere Kommunikation (z. B. bzgl. Hygiene/Abstand, Sorgen/Ängste vor Ansteckung) }\end{array}$ \\
\hline Visiten $(\mathrm{N}=9)$ & Visiten wurden in kleinerer Gruppe durchgeführt, Aussetzen der Chefarztvisite, Visiten unter erhöhten Sicherheitskautelen \\
\hline $\begin{array}{l}\text { Einführung von Screenings } \\
(\mathrm{N}=8)\end{array}$ & Testung bei Patientinnen und Patienten bei Aufnahme und im Verlauf bei Bedarf \\
\hline $\begin{array}{l}\text { Stationen und Zimmer } \\
(\mathrm{N}=7)\end{array}$ & $\begin{array}{l}\text { Teilweise Schließung von Stationen, Schaffung von Einzelzimmern, Umrüsten von Zimmern und Verteilung von Zimmern } \\
\text { über Abteilungen hinweg (Außenlieger) }\end{array}$ \\
\hline
\end{tabular}

Stationen komplett geschlossen oder an andere Kliniken abgegeben. Acht (von 34; 23,5\%) leitende Ärztinnen und Ärzte beschrieben, dass elektive Operationen zugunsten der Fokussierung auf operative Eingriffe von Notfällen und von Tumorpatientinnen und -patienten verschoben oder abgesagt wurden. Vier (von 34; 11,8\%) leitende Ärztinnen und Ärzte gaben an, dass die Belegung zum Zeitpunkt der Befragung wieder anzusteigen begann bzw. die Volllast bereits fast wieder erreicht worden ist. Im Gegensatz zu den oben beschriebenen Veränderungen berichteten leitende Ärztinnen und Ärzten aus drei (von 34; 8,8\%) Kliniken durchweg „Normalbetrieb“ hinsichtlich der Belegung.

Mit Blick auf die Auslastung gestaltete sich die Situation in den Ambulanzen ( $n=37$ ) sehr ähnlich. In einigen Kliniken kam es zu einer Reduktion um bis zu $90 \%$. Zum einen sagten Patientinnen und Patienten aus Sorge vor einer möglichen Ansteckung ambulante Termine ab, zum anderen verschoben die Ambulanzen nichtdringliche Termine. Soweit möglich, wurden Nachsorgetermine telefonisch angeboten bzw. umgesetzt. Einige leitende Ärztinnen und Ärzte äußerten die Befürchtung, dass Patientinnen und Patienten ohne COVID-19-Erkrankung durch die Einschränkungen vernachlässigt werden könnten. In einigen Ambulanzen entstanden kumulativ lange Wartelisten. Die aktuelle Situation in den Tageskliniken ( $n=17)$ ähnelt dem beschriebenen Bild.

\section{Patientenzusammensetzung}

Die Mehrheit der leitenden Ärztinnen und Ärzte berichtete von Veränderungen in der Patientenzusammensetzung, die stationär behandelt wurden ( $n=27$ von 34; 79,4\%). In den Kliniken wurden größtenteils nur noch Notfälle und schwer- bzw. schwerstkranke Menschen behandelt. Fünf (von 34; 14,7\%) leitende Ärztinnen und Ärzte gaben an, keine Veränderungen in der Zusammensetzung beobachtet zu haben. Im ambulanten Bereich sprachen 23 (von 37; $62,2 \%$ ) von Veränderungen in der Patientenpopulation. Auch hier wurden fast ausschließlich Notfälle und schwere Erkrankungen (weiter) behandelt. Sieben (von 37; 18,9\%) Befragte konnten keine signifikanten Veränderungen feststellen. Acht (von 17; 47,1\%) machten für ihre Tageskliniken dazu Angaben, fünf (von 8; 62,5\%) von ihnen sahen keine Veränderungen. Die übrigen drei (von 8; $37,5 \%$ ) gaben an, aktuell lediglich schwer erkrankte Patientinnen und Patienten zu sehen.

\section{Klinische und organisatorische Prozesse}

- Tab. 2 zeigt die Auswirkungen auf klinische und organisatorische Prozesse im stationären Bereich. Sie beziehen sich v. a. auf die Kommunikation (z. B. Reduktion von Besprechungen, Umstellung auf Videokonferenzen), die Organisation des Personals (z. B. neue Teamstrukturen) und für Patientinnen und Patienten (v. a. Besuchsund Hygieneregeln) sowie die Visitendurchführung und die Umsetzung von notwendigen Screeningmaßnahmen.

Auch im ambulanten und tagesklinischen Bereich wurden klinische und organisatorische Prozesse angepasst. Es wurden z. B. ebenfalls kleinere Teams gebildet, feste Räume vergeben und $\mathrm{Vi}$ deokonferenzen durchgeführt. Wartebereiche wurden umgebaut, Wartezeiten verkürzt und Termine gestreckt, um Abstandsregeln zu beachten und Kontakte einzuschränken.

\section{Diagnostik und Behandlung}

28 von 34 Befragten (82,4\%) machten Angaben zu Veränderungen der diagnostischen und therapeutischen Angebote, wobei 11 (von 34; 32,4\%) angaben, dass es im stationären Bereich keine relevanten Auswirkungen gab. Drei (von 34; 8,8\%) Befragte berichteten von eingeschränkter Diagnostik, zwei (von 34; 5,9\%) beschrieben diese als unverändert und eine (von 34; 2,9\%) Person nannte eine Steigerung der diagnostischen Maßnahmen. Darüber hinaus wurde die telefonische Nachsorge als ein verändertes Behandlungsangebot genannt. Im ambulanten und tagesklinischen Bereich wurden vereinzelt Jahreskontroll- und Nachsorgeuntersuchungen, Aufklärungsgespräche und Diagnosemitteilungen verstärkt telefonisch oder per Video durchgeführt.

\section{Präventive, therapeutische und Nachsorgebedarfe sowie neue Behandlungsangebote}

Alle leitenden Ärztinnen und Ärzte berichteten, dass Regelungen und Vorkehrungen getroffen werden mussten, um den Präventionsund Behandlungs- sowie Nachsorgebedarf der Nicht-Corona-Betroffenen adäquat abzudecken. Die Befragten berichteten v. a. von Vorkehrungen zur Kontaktverringerung (35 Nennungen), dem Einsatz von Schutzkleidung (18 Nennungen) sowie der Einführung von Screening-Maßnahmen (12 Nennungen) und einer Verstärkung der Hygienemaßnahmen (5 Nennungen).

$44,7 \%$ (17 von 38) der Befragten gab an, dass ihre Klinik im Zuge der Pandemie neue Behandlungsangebote, wie z. B. Tele- oder 
- Tab. 3 Auswirkungen auf das Personal

\begin{tabular}{|c|c|}
\hline Kategorien & Subkategorien \\
\hline $\begin{array}{l}\text { Negative emotionale } \\
\text { Auswirkungen }\left(\mathrm{N}^{*}=25\right)\end{array}$ & $\begin{array}{l}\text { Verunsicherung, Angst, gedrückte Stimmung, Gefährdungsgefühl, Wunsch nach Normalität, Widerstände beim } \\
\text { Hochfahren der Versorgung, Sorgen, Stresserleben, Unsicherheiten bei Anweisungen }\end{array}$ \\
\hline $\begin{array}{l}\text { Positive emotionale } \\
\text { Auswirkungen }(\mathrm{N}=20)\end{array}$ & Solidarität, Rücksichtnahme, Gelassenheit, Optimismus, Teamgeist, hohe Motivation, Besonnenheit, Pragmatismus \\
\hline \multirow[t]{2}{*}{ Arbeitsaufwände $(\mathrm{N}=11)$} & $\begin{array}{l}\text { Einerseits: Reduktion von Aufwänden und der Mehrfachbelastung, Abbau von Überstunden, mehr Zeit für } \\
\text { Einarbeitung von neuem Personal, Freiräume für wissenschaftliches Arbeiten }\end{array}$ \\
\hline & $\begin{array}{l}\text { Andererseits: erhöhter Kommunikationsaufwand, teilweise verlängerte Schichten bei Ärzten, sehr hohe Entschei- } \\
\text { dungsdichte, schlechtere (chirurgische) Einarbeitung bzw. Weiterbildung }\end{array}$ \\
\hline Home Office $(\mathrm{N}=5)$ & $\begin{array}{l}\text { Home-Office-Möglichkeit ist erfolgreich, entspricht verstärktem Wunsch bei Mitarbeitenden; jedoch auch } \\
\text { Einschätzung, dass Home Office nicht für alle geeignet ist }\end{array}$ \\
\hline
\end{tabular}

Video-Sprechstunden, eingeführt hat. Weitere 21,1 \% (8 von 38) berichteten, dass die Klinik bereits bestehende Angebote der digitalen und Telekommunikation stärker nutzt als vor der Pandemie. Diejenigen, die neue Angebote eingesetzt haben oder bestehende Angebote stärker nutzen ( $n=25 ; 65,8 \%$ ), beschrieben deren Akzeptanz bei den Patientinnen und Patienten größtenteils als „gut“ bis „sehr gut“ ( $n=18$ von 25; $72 \%$ ). 15 von 25 (60\%) bewerteten den Einsatz dieser als „erfolgreich“ bis „sehr erfolgreich“.

\section{Arbeitsprozesse, Personal und Mitarbeiterschutz sowie interdisziplinäre Zusammenarbeit}

\section{Arbeitsprozesse}

Fast alle leitenden Ärztinnen und Ärzte (36 von 38; 94,7 \%) berichteten von relevanten Auswirkungen auf die Arbeitsprozesse in ihrer Klinik unter der Pandemie. Dabei nannten die Befragten v. a. die Themen Kommunikation, Personalangelegenheiten und den allgemeinen Arbeitsaufwand. Insgesamt hätten die interne Kommunikation und der Austausch eher abgenommen, die Kommunikationsprozesse gestalteten sich aufwendiger. Beim Personal gab es insbesondere Hinweise, wie „fehlende Auslastung“ oder „Überkapazitäten“, „Einrichtung von parallelen Teams“ und die „Verlegung von Personal ins Home-Office“. Einige Befragte beschrieben, dass es einerseits „weniger Hektik“ und ,je nach Bereich weniger Aufwand“ gegeben habe, andererseits „Mehraufwände durch Bürokratie“ entstanden seien und dass die „Arbeit langsamer und zeitaufwändiger“ von statten ginge sowie der „logistische Aufwand“ gestiegen sei.

Personal und Mitarbeiterschutz

94,7\% (36 von 38) der leitenden Ärztinnen und Ärzten berichtete von Auswirkungen auf das Verhalten und Erleben ihrer Mitarbeiterinnen und Mitarbeitern unter der Pandemie ( $>$ Tab. 3), insbesondere positive wie negative emotionale Auswirkungen sowie veränderte Arbeitsaufwände.

63,2\% (24 von 38) der leitenden Ärztinnen und Ärzten sprachen auch von Defiziten oder Engpässen mit Blick auf die Schutzmaßnahmen für Mitarbeiterinnen und Mitarbeiter, v. a. zu Beginn der Krise. Das betraf v. a. Engpässe beim Schutzmaterial, wie z. B. sichere Masken (FFP-2, FFP-3), Desinfektionsmittel und Schutzausrüstung im Allgemeinen. Die meisten Engpässe wurden jedoch als temporär und mittlerweile als behoben beschrieben.
Interdisziplinäre Zusammenarbeit

86,8 \% (33 von 37 der leitenden Ärztinnen und Ärzten beschrieb Veränderungen in der interdisziplinären Zusammenarbeit in ihrer Klinik. Es gab 18 Nennungen, die positive bis sehr positive Auswirkungen beschreiben, wie z. B. einen deutlichen Anstieg an interdisziplinärer Zusammenarbeit, eine effizientere Gestaltung der Zusammenarbeit (z. B. durch digitale Lösungen), eine erhöhte Teilnehmerzahl klinikinterner Besprechungen, eine große Solidarität und ein gestärktes Zusammengehörigkeitsgefühl. 14 Nennungen sahen eher problematische bis sehr negative Veränderungen, wie z. B. weniger Interaktion und Kontakte, das Vermissen persönlicher Meetings, der Wegfall von Fortbildungen, eine aufwändigere Kommunikation (via Video u. ä.) bis hin zum kompletten Verlust der Zusammenarbeit.

\section{Diskussion}

Sowohl die COVID-19-Pandemie an sich als auch die Implementierung der dadurch notwendigen Maßnahmen bedeuteten eine große Anpassungsleistung für alle Einrichtungen der Gesundheitsversorgung, insbesondere auch für die Universitätskliniken $[9,15,16]$.

Die berichteten Ergebnisse dokumentieren, was die von der (Gesundheits-)Politik vorgegebenen Maßnahmen konkret für einen Maximalversorger bedeuten, u. a. bezogen auf die Bettenbelegung, die Veränderung des Operationsgeschehens, personelle und organisatorische Veränderungen inklusive das Erleben des Personals sowie die Umsetzung präventiver Maßnahmen. Indirekt davon waren auch zahlreiche Interaktionsprozesse sowie die Wahrung von Patientenrechten tangiert. Wie gravierend diese Umstellungen ausfielen, zeigen Ergebnisse bzgl. der Arbeitsaufwände, z. B. durch die Anpassung von Arbeitsabläufen, die erhöhten Kommunikationsbedarfe und die Einführung von zusätzlich notwendigen Hygienemaßnahmen. Besonders zu Beginn der Pandemie führten Engpässe in der Beschaffung von Schutzkleidung zu stark erhöhten Zusatzaufwänden. Das Personal ist sowohl durch die Pandemie an sich als auch durch die damit verbundenen Maßnahmen belastet.

Die umgesetzten Maßnahmen führten in den meisten Bereichen zu einer geringeren Auslastung des Klinikums. Die dadurch im Jahr 2020 zu erwartenden und durch Hochrechnungen bestätigten Verluste stehen bisher noch nicht im Fokus der Diskussion. Es ist aber jetzt schon davon auszugehen, dass die vom BMG zugesprochenen Ausfallfinanzierungen nicht ausreichen werden, die zusätzlich ent- 
standenen Bedarfe von Universitätskliniken zu decken. Es bedarf daher in den nächsten Wochen und Monaten Lösungsmöglichkeiten, die auf der politischen Ebene gefunden werden müssen.

Wenn man bedenkt, wie schwierig es normalerweise ist, Veränderungsprozesse in einem großen Klinikum umzusetzen, ist dies insgesamt effizient und konfliktarm gelungen. Viele der beschriebenen gravierenden Eingriffe in Arbeitsabläufe, die Ressourcenverteilung oder die Patienten- und Mitarbeiterrechte waren nur möglich bzw. durchsetzbar, weil die große Mehrheit der beteiligten Mitarbeiterinnen und Mitarbeiter sowie der Patientinnen und Patienten die unmittelbare Notwendigkeit mitgetragen hat. Das Fortbestehen dieser Bereitschaft über längere Zeit kann aber keineswegs als selbstverständlich vorausgesetzt werden. Die Wiederherstellung der Normalität im UKE könnte vergleichbare Diskussionsprozesse auslösen, wie wir sie auf gesellschaftlicher Ebene bei der Aufhebung des „Lockdown“ derzeit erleben.

Auch in dieser Krise sollte die Frage gestellt werden, welche der „zwangsweise“ eingetretenen Veränderungen neue Chancen für die Zukunft bieten. Hier sind insbesondere z. B. die Digitalisierung von Arbeits- und Kommunikationsprozessen, die verstärkte Nutzung von Home-Office-Möglichkeiten und die Beschleunigung von Entscheidungs- und administrativen Prozessen zu diskutieren.

Die hier berichteten Ergebnisse beruhen auf einer Studie, die kurzfristig realisiert werden musste. Die Ergebnisse und ihre Reflektion beziehen sich auf Beobachtungen eines aktuellen Status quo inmitten der COVID-19-Krise, die längerfristigen Entwicklungen und Herausforderungen sind erst mittelfristig abzuschätzen und erfordern weitere Verlaufsuntersuchungen.

Mit der Studie ist aber auch etwas Seltenes gelungen, nämlich eine vollständige Stichprobe von fachkundigen Expertinnen und Experten zeitnah zu gewinnen, sodass das gesamte medizinische Spektrum und die Besonderheiten der unterschiedlichen Fächer eines großen Universitätsklinikums Berücksichtigung finden konnten. Ein Grund war der Umstand, dass sich die Ärzte und Ärztinnen Sorge um Patientinnen und Patienten machten, deren Behandlung deutlich eingeschränkt worden ist. Die Identifikation der Befragten mit dem UKE, die Unterstützung der Studie durch den UKE-Vorstand und die Tatsache, dass die vier Interviewer professorale Kollegen sind, könnten die Ergebnisse ungewollt in Richtung einer positiven Selbstdarstellung beeinflusst haben.

Da nur eine einzige Klinik und diese noch aus dem speziellen Versorgungsbereich der Hochschulmedizin Berücksichtigung finden konnte, bleibt die Frage der Generalisierung der Befunde offen. Darüber hinaus ist darauf hinzuweisen, dass die Ergebnisse ausschließlich die Perspektive des leitenden ärztlichen Personals wiedergeben. Einzelne Aspekte könnten sich z. B. aus der Sicht des Pflegepersonals durchaus anders darstellen. Nichtsdestotrotz glauben wir, dass die Befragungsergebnisse nicht nur für den Standort Hamburg, sondern auch für andere Universitätskliniken und andere Gesundheitseinrichtungen informativ sein können. Es wird deutlich, dass die Pandemie einen Maximalversorger extrem fordert. Folglich helfen die Ergebnisse, die Auswirkungen der COVID-19-Pandemie auf die Versorgung, Arbeitsprozesse und Mitarbeitenden noch besser zu verstehen. Zudem können sie dabei unterstützen, zukünftig Maßnahmen für pandemische Krisensituationen besser zu beschreiben und bestehende Regelungen anzupassen.

\section{Danksagung}

Wir bedanken uns beim Vorstand und den Kolleginnen und Kollegen des UKE für das Engagement in der Phase besonderer klinischer und organisatorischer Herausforderungen.

\section{Interessenkonflikt}

Alle Autoren sind am UKE beschäftigt. Die vier Interviewer sind professorale Kollegen der Befragten.

\section{Literatur}

[1] Sohrabi C, Alsafi Z, O'Neill N et al. World Health Organization declares global emergency: A review of the 2019 novel coronavirus (COVID-19). International Journal of Surgery 2020; 71: 71-76

[2] Stefanini GG, Azzolini E, Condorelli G. Critical organizational issues for cardiologists in the COVID-19 outbreak: a frontline experience from Milan, Italy. Circulation 2020; 141: 1597-1599

[3] Repici A, Maselli R, Colombo M et al. Coronavirus (COVID-19) outbreak: what the department of endoscopy should know. Gastrointestinal Endoscopy 2020; [in press]

[4] Bundesministerium für Gesundheit Coronavirus SARS-CoV-2: Chronik der bisherigen Maßnahmen. Im Internet: https://www.bundesgesundheitsministerium.de/coronavirus/chronik-coronavirus.html; Stand: 08.05.2020

[5] Collaborative CovidSurg, Nepogodiev D, Bhangu A. Elective surgery cancellations due to the COVID-19 pandemic: global predictive modelling to inform surgical recovery plans. British Journal of Surgery. 2020; [in press]

[6] Beerheide R, Osterloh F. Coronakrise: Acht Gesetze in acht Tagen. Deutsches Ärzteblatt 2020; A695-A697

[7] Bundesministerium für Gesundheit. Referentenentwurf einer Verordnung zur Aufrechterhaltung und Sicherung intensivmedizinischer Krankenhauskapazitäten (DIVI Intensivregister-Verordnung). 2020

[8] Universitätsklinikum Hamburg-Eppendorf. Informationen zum Corona-Virus. 2020; Im Internet: https://www.uke.de/corona-virus/ index.html; Stand: 08.05.2020

[9] Waldman G, Mayeux R, Claassen J et al. Preparing a neurology department for SARS-CoV-2 (COVID-19): Early experiences at Columbia University Irving Medical Center and the New York Presbyterian Hospital in New York City. Neurology 2020; 94: 886-891

[10] Universitätsklinikum Hamburg-Eppendorf. Geschäftsbericht und Jahrbuch 2018 - Der Zukunftsplan 2050 wird Realität. 2019

[11] Robert Koch-Institut. Im Internet: https://experience.arcgis.com/exper ience/478220a4c454480e823b17327b2bf1d4/page/page_1/ Stand: 20.05.2020

[12] Behörde für Gesundheit und Verbraucherschutz. Informationen zum aktuellen Stand COVID-19 in Hamburg. Im Internet: https://www. hamburg.de/coronavirus/pressemeldungen/13889420/202004-30-coronavius-aktueller-stand/; Stand: 20.05.2020

[13] Proctor E, Silmere $\mathrm{H}$, Raghavan R et al. Outcomes for implementation research: conceptual distinctions, measurement challenges, and research agenda. Administration and Policy in Mental Health and Mental Health Services Research 2011; 38: 65-76

[14] Mayring P. Qualitative Inhaltsanalyse - Grundlagen und Techniken. 2010; Weinheim: Beltz

[15] Wong J, Goh QY, Tan Z et al. Preparing for a COVID-19 pandemic: a review of operating room outbreak response measures in a large tertiary hospital in Singapore. Canadian. Journal of Anesthesia 2020; 67: 1-14

[16] Adams JG, Walls RM. Supporting the health care workforce during the COVID-19 global epidemic. JAMA 2020; 323: 1439-1440 